\title{
A study of Traveling Ionospheric Disturbances and Atmospheric Gravity Waves using EISCAT Svalbard Radar IPY-data
}

\author{
A. Vlasov ${ }^{1,2, *}$, K. Kauristie ${ }^{1}$, M. van de Kamp ${ }^{1}$, J.-P. Luntama ${ }^{1,3}$, and A. Pogoreltsev ${ }^{2}$ \\ ${ }^{1}$ Finnish Meteorological Institute, Helsinki, Finland \\ ${ }^{2}$ Russian State Hydrometeorological University, Weather Forecasts Department, Saint-Petersburg, Russia \\ ${ }^{3}$ SSA-SWE Segment, ESAC, Spain \\ " now at: Karlsruhe Institute of Technology, Institute for Meteorology and Climate Research, Karlsruhe, Germany
}

Received: 3 October 2010 - Revised: 22 July 2011 - Accepted: 3 October 2011 - Published: 24 November 2011

\begin{abstract}
We present a statistical study of Traveling Ionospheric Disturbances (TIDs) as observed by the EISCAT Svalbard Radar (ESR) during the continuous IPY-run (March 2007-February 2008) with field-aligned measurements. We have developed a semi-automatic routine for searching and extracting Atmospheric Gravity Wave (AGW) activity. The collected data shows that AGW-TID signatures are common in the high-latitude ionosphere especially in the field-aligned ion velocity data (244 cases of AGW-TID signatures in daily records), but they can be observed also in electron density (26 cases), electron temperature (12 cases) and ion temperature (26 cases). During the IPY campaign (in solar minimum conditions) AGW-TID events appear more frequently during summer months than during the winter months. It remains still as a topic for future studies whether the observed seasonal variation is natural or caused by seasonal variation in the performance of the observational method that we use (AGW-TID signature may be more pronounced in a dense ionosphere). In our AGW-TID dataset the distribution of the oscillation periods has two peaks, one around $0.5-0.7 \mathrm{~h}$ and the other around $1.1-1.3 \mathrm{~h}$. The diurnal occurrence rate has a deep minimum in the region of magnetic midnight, which might be partly explained by irregular auroral activity obscuring the TID signatures from our detection routines. As both the period and horizontal phase speed estimates (as derived from the classical AGW dispersion relation) show values typical both for large scale TIDs and mesoscale TIDs it is difficult to distinguish whether the generator for highlatitude AGW-TIDs resides typically in the troposphere or in the near-Earth space. The results of our statistical analysis give anyway some valuable reference information for the future efforts to learn more about the dominating TID source
\end{abstract}

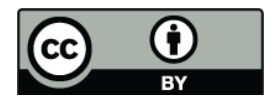

Correspondence to: A. Vlasov (avlasov@rshu.ru) mechanisms in polar cap conditions, and to improve AGW simulations.

Keywords. Ionosphere (Ionosphere-atmosphere interactions; Polar ionosphere) - Meteorology and atmospheric dynamics (Waves and tides)

\section{Introduction}

Observations by ionospheric radars and ionosonde networks have revealed that large scale wave-like structures can propagate in the ionosphere for long distances, e.g. from highlatitudes to mid-latitudes or even to the equatorial region (Hunsucker, 1982). The mechanism responsible for generation of those Travelling Ionospheric Disturbances (TIDs) was originally suggested in the pioneering study by Hines (1960) and usually referred to as Atmospheric Gravity Waves (AGWs), whose dynamics is mostly defined by the interplay of buoyancy and gravitational forces (hereafter such TIDs will be referred as AGW-TID). AGW-TIDs have time periods from some tens of minutes up to approximately $3 \mathrm{~h}$, their wavelenghts are typically longer than $100 \mathrm{~km}$, and they can be grouped into two categories: Large scale TIDs with horizontal scale lenghts above $1000 \mathrm{~km}$ and periods in the range of $0.5-3 \mathrm{~h}(30-180 \mathrm{~min})$ and medium scale TIDs with horizontal wavelengths of several hundreds of $\mathrm{km}$ and oscillation periods typically between $0.2-1 \mathrm{~h}(12-60 \mathrm{~min})$ (Hunsucker, 1982; Hocke and Schlegel, 1996). The horizontal propagation speeds of large-scale AGW-TIDs is comparable with the speed of sound in the thermosphere, i.e. from $\sim 400 \mathrm{~m} \mathrm{~s}^{-1}$ to $1000 \mathrm{~m} \mathrm{~s}^{-1}$. Medium scale TIDs propagate at slower speeds, typicaly in the range from 100 to $250 \mathrm{~m} \mathrm{~s}^{-1}$. Currently the general understanding is that meso-scale AGW-TIDs may have their origin both in the lower atmosphere and in the upper atmosphere (Viereck, 1991), while large-scale TIDs

Published by Copernicus Publications on behalf of the European Geosciences Union. 
result from magnetosphere-ionosphere coupling phenomena (Hunsucker, 1982).

TIDs and their connection with AGWs in the upper part of the atmosphere have been investigated intensively since the 1950s. The review paper by Hocke and Schlegel (1996) gives a comprehensive picture of the advancements in the observational, theoretical and simulation work conducted in this field. Physical mechanisms responsible for generation of TIDs types and methodology to estimate AGW characteristics from TID observations have been some of the most important issues. From the basis of sophisticated simulation runs Kirchengast (1997) has shown how TIDs of space origin are distinguished from AGW-TIDs in the context of Incoherent Scatter Radar (ISR) observations of ionospheric electron density, electron and ion temperatures and ion velocity. ISR data was also used for estimation of the horizontal and vertical wavelengths of the thermospheric neutral wind oscillations (Lanchester et al., 1993).

There have been a number of studies addressing the climatology of TIDs based on the data from networks of groundbased GPS receivers located in Japan (Saito et al., 1998; Tsugawa et al., 2004), Southern California (Kotake et al., 2007) and a larger network covering North America (Tsugawa et al., 2007). In these studies the diurnal and annual variability of periods, horizontal wavelengths, horizontal phase velocities and amplitudes of TIDs together with their connection to geomagnetic activity were investigated using maps of differential TEC (Total Electron Content). The studies have confirmed that occurrence of Large-Scale TIDs (LSTIDs) correlates very well with periods of high geomagnetic activity, while Medium-Scale TIDs (MSTID) tend to be quiettime events. Tsugawa et al. (2004) have studied the appearance of TIDs in Japan during solar maximun conditions (years 1999-2002). In their dataset only $28 \%$ of the LSTIDs took place during $K_{\mathrm{p}} \leq 3$. In such conditions the seasonal distribution of LSTID activity shows maxima around the vernal and autumnal equinoxes, thus they follow nicely the occurrence distribution of strong geomagnetic disturbances as controlled by the Russell-McPherron effect (Russel and McPherron, 1973). Kotake et al. (2007) categorised MSTIDs into three classes: (1) daytime MSTIDs, being clearly an ionospheric manifestation of AGW activity as described by Hines (1960); (2) night-time MSTIDs, possibly caused by electrodynamical phenomena, and (3) dusk MSTIDs, generated by the moving solar terminator. Nighttime MSTIDs have a maximum occurrence rate during summer while daytime MSTIDs occur more frequently during winter.

While the appearance of AGWs at auroral and midlatitudes have been studied comprehensively with various observational and modelling works, the appearance of AGWTIDs in the polar cap area is a less addressed topic. So far only the study by Bristow et al. (1996) is known to us, where a higher occurrence of AGW-TID activity during winter months was reported based on SuperDARN HF radar observations of AGW signatures in the ground-backscattered signal. Data as recorded by some SuperDARN radars located in the Northern Hemisphere show roughly double the AGW occurrence rates during winter months of those during summer months in the years 1993-1995. Bristow et al. (1996) proposed that this seasonal variability is caused by a variation of vertical temperature gradient at mesopause altitudes, which controls AGW propagation and hence their occurrence at F-layer altitudes which SuperDARN radars monitor.

The main goal of this paper is to contribute to the knowledge of AGW-TID in the high latitude ionosphere (in the polar cap and at the poleward boundary of the auroral oval). Here we present statistics of AGW-TID events observed with the EISCAT Svalbard Radar (ESR) during the one-year run conducted as part of the fourth International Polar Year programme (2007-2009). We have developed a search method, which picks only TIDs which show a phase delay in altitude (a downward propagating phase front). In several previously published AGW studies utilizing Incoherent Scatter Radar (ISR) measurements, such a behavior has been considered as the key feature for those TIDs where AGWs vary the ionospheric conditions. As the IPY run was conducted during a solar minimum period, we can anticipate that ionospheric signatures of AGW activity might be less obscured by other more irregular geospace activity. According to our knowledge this is the first study when such a big ISR dataset has been used in statistical analysis of AGW-TIDs.

In the following, Sect. 2 introduces our data analysis methods, Sect. 3 presents the main results with some discussion and Sect. 4 summarises the main findings of this paper.

\section{Data and analysis methods}

\subsection{Dataset description}

Measurements with Incoherent Scatter Radars (ISRs) allow derivation of some basic ionospheric plasma parameters: electron density $\left(N_{\mathrm{e}}\right)$, electron temperature $\left(T_{\mathrm{e}}\right)$, ion temperature $\left(T_{\mathrm{i}}\right)$ and ion drift velocity $\left(V_{\mathrm{i}}\right)$. Incoherent backscatter comes from electrons whose motion is controlled by thermal fluctuations of the more massive ions. The dominating modes in these fluctuations are ion acoustic waves and Langmuir waves (Hagfors, 1961). As the backscattered signal is extremely weak, powerful transmitters are required, and for economical reasons measurements are conducted usually as short campaigns. During the recent International Polar Year (IPY), however, a long almost continuous ISR run was conducted with the EISCAT Svalbard Radar (ESR) (Wannberg et al., 1997) lasting from the beginning of March 2007 to the end of February 2008. The measurements were made with the $42 \mathrm{~m}$ dish antenna located at $78^{\circ} 09^{\prime} 11^{\prime \prime} \mathrm{N}, 16^{\circ} 01^{\prime} 44^{\prime \prime} \mathrm{E}$ which has a fixed pointing direction along the geomagnetic field line (in Svalbard the dip angle is $82^{\circ} 06^{\prime}$ ). ESR measurements cover around $64 \%$ of the total time within the IPY campaign. More detailed information about the data 
coverage is given in Fig. 1. The relatively long breaks in the measurements in May, September and November are caused by radar malfunctions or problems in the power supply for the radar. The processed data are time series of electron density, electron temperature, ion temperature and line-of-sight ion drift velocity in an altitude domain of approximately 90 $450 \mathrm{~km}$. The time resolution is $1 \mathrm{~min}$. The resolution in altitude varies: increasing from $\sim 3 \mathrm{~km}$ to $\sim 5 \mathrm{~km}$ in the E-layer and from $\sim 7 \mathrm{~km}$ to $\sim 22 \mathrm{~km}$ in the F-layer. Vertical profiles contain 32 data points (11 in the E-layer and 21 in the Flayer).

\subsection{Ionospheric response to AGW}

AGW are semiperiodic motions of air accompanied by compression/rarefaction (Hines, 1960), and their signatures can be observed in all ISR parameters through interaction with ionospheric plasma (Kirchengast, 1996). In a field-aligned observation AGW-related TIDs appear typically as downward propagating phase fronts, and the signatures are often most pronounced in ion velocity or electron density data (Nygren et al., 1992). Downward propagating phase fronts are seen in the ISR beam for waves coming from a remote source, because wave propagation speed increases with altitude (Hines, 1960). This is due to the fact that the wave fronts propagating at higher altitudes reach the radar beam earlier than those propagating at lower altitudes.

The simulation results by Kirchengast (1997) address several different generation mechanisms of TID: the AGW, periodic fluctuations in the $\boldsymbol{E} \times \boldsymbol{B}$ plasma drift and periodic fluctuations in the auroral precipitation. In all of these cases different physical and chemical processes transferring mass, momentum and energy between the charged and neutral gas populations may have different impact, and consequently the TID signatures in the ISR data vary from case to case. In the case of AGW, where the original cause for fluctuations comes from processes in the much more abundant neutral gas, the TIDs can be considered as passive tracers reflecting the AGW charateristics.

In order to efficiently distinguish between AGW-TIDs and TIDs which are caused by periodic variations in different magnetosphere-ionosphere coupling phenomena (which are global and thus affecting also the ionospheric conditions just above the observing point) (Kirchengast, 1997) we have formulated a set of criteria for our search routines:

1. AGW-TID are quasi-periodic undulations with periods from tens of minutes to a couple of hours (Hunsucker, 1987);

2. AGW-TID signatures cover the whole F- and sometimes the E-layer (Williams, 1989; Djuth et al., 1997);

3. AGWs are best seen in line-of-the-sight ion velocity data (Williams, 1989);

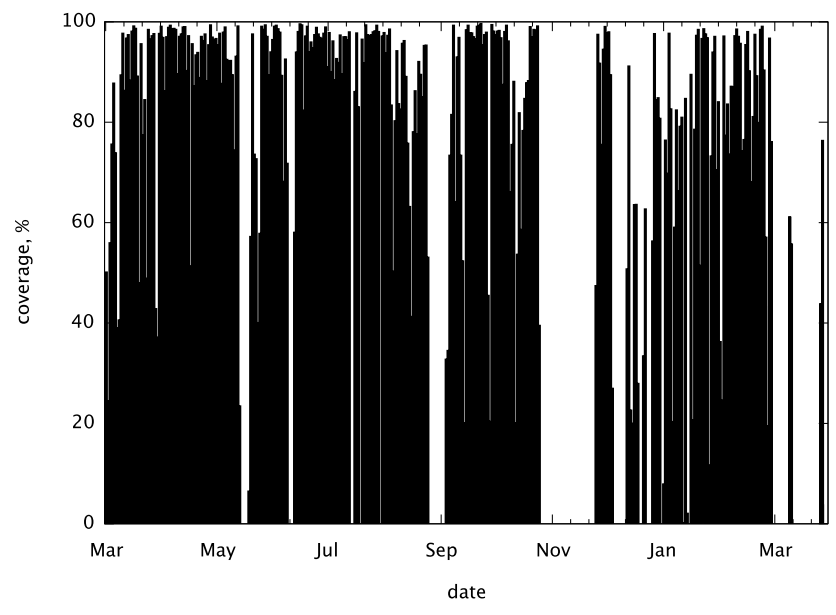

Fig. 1. The fraction of time inside a day covered by observation within the IPY-run.

4. AGW-TID demonstrate "downward" phase propagation in time (Kirchengast, 1997; Williams, 1989).

According to the simulation results by Kirchengast (1997) especially the fourth criterion is characteristic for the AGWTIDs, which were discussed in the pioneering article by Hines (1960). In order to optimise the search and extraction of AGW-TID we have developed a set of automatic routines, which search for data samples that fulfil criteria No 1 (presence of quasi-periodic disturbances, with periods within certain ranges) and No 2 (high coherence of quasi-periodic disturbances over extended range of altitudes) mentioned above. The input for the automated part is the ESR-IPY data as described in Sect. 2.1. The check for the other two criteria and the final decision is made by visual analysis. The flow-chart of the automated part of the analysis is presented in Fig. 2, and individual steps are described in the next subsections.

\subsection{Spectral analysis}

The traditional way to extract AGW-TID from ISRmeasurements is to utilize digital filters (Hocke et al., 1996). In order to choose the correct passband for the filter we first apply spectral analysis to the daily ISR data files. We have chosen to use the Lomb-Scargle periodogram approach (Press and Teukolsky, 1988) in the analysis because it can treat unevenly sampled data and thus avoids the interpolation to cover the data gaps (in time). The method provides the least-square fit of the data to a combination of sine and cosine functions. This combination can then be used for the gap filling (Hocke, 1998; Hocke and Kämpfer, 2008).

Following Press and Teukolsky (1988), the normalised Lomb-Scargle periodogram (spectral power as a function of angular frequency $\omega$ ) for a time series $h_{i}=h\left(t_{i}\right), i=1, \ldots, N$ is defined as 


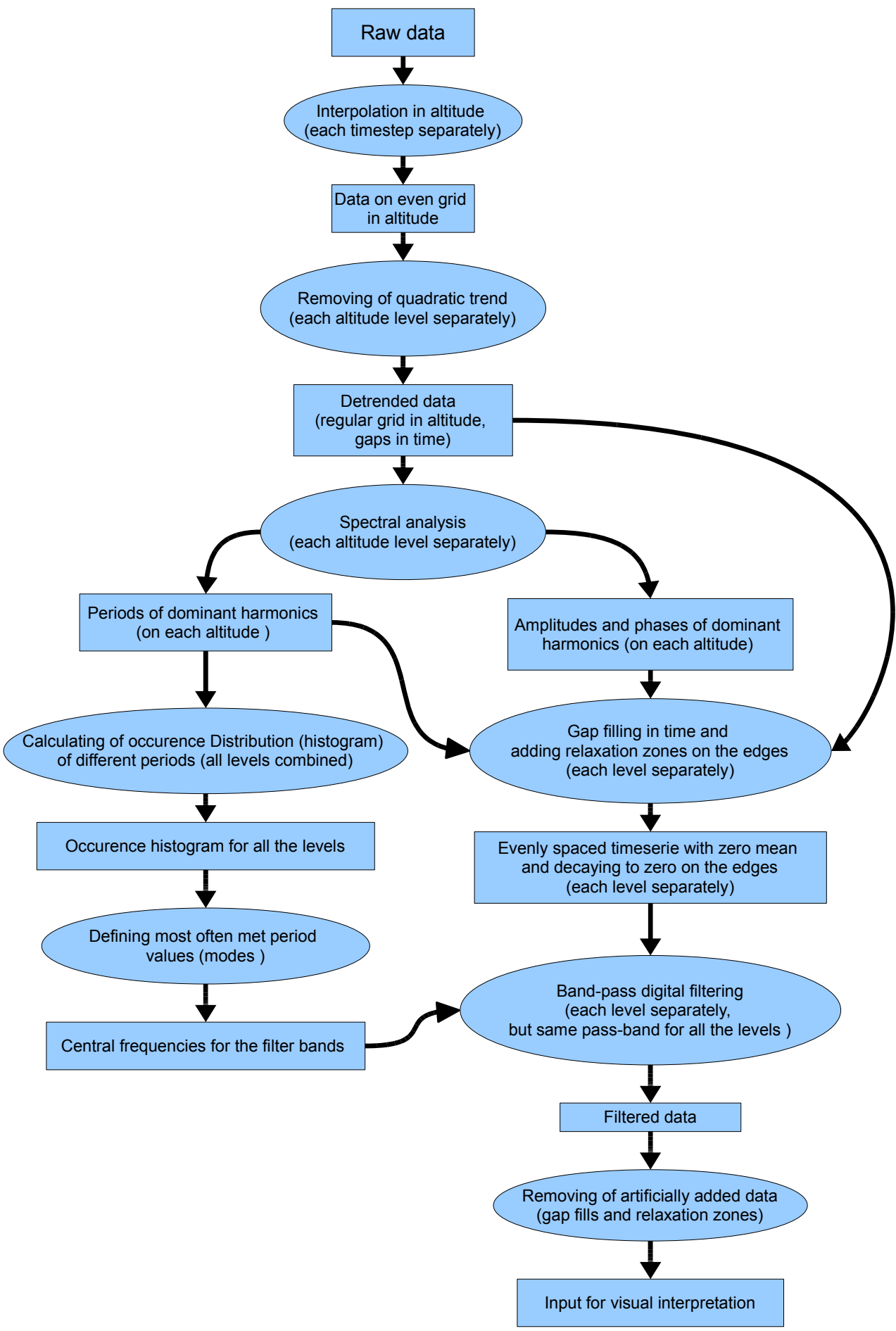

Fig. 2. The flow-chart of the automatic part of analysis. Software modules are represented by ovals. Rectangular boxes describe the input/output data of individual software modules.

$$
\begin{aligned}
P_{N}(\omega)= & \frac{1}{2 \sigma^{2}}\left\{\frac{\left[\sum_{i}\left(h_{i}-\bar{h}\right) \cos \omega\left(t_{i}-\tau\right)\right]^{2}}{\sum_{i} \cos ^{2} \omega\left(t_{i}-\tau\right)}\right. \\
& \left.+\frac{\left[\sum_{i}\left(h_{i}-\bar{h}\right) \sin \omega\left(t_{i}-\tau\right)\right]^{2}}{\sum_{i} \sin ^{2} \omega\left(t_{i}-\tau\right)}\right\}
\end{aligned}
$$

where $\tau$ is defined from

$\tan (2 \omega \tau)=\frac{\sum_{i} \sin 2 \omega t_{i}}{\sum_{i} \cos 2 \omega t_{i}}$

and $\bar{h}$ and $\sigma^{2}$ are the mean value and variance, respectively. 


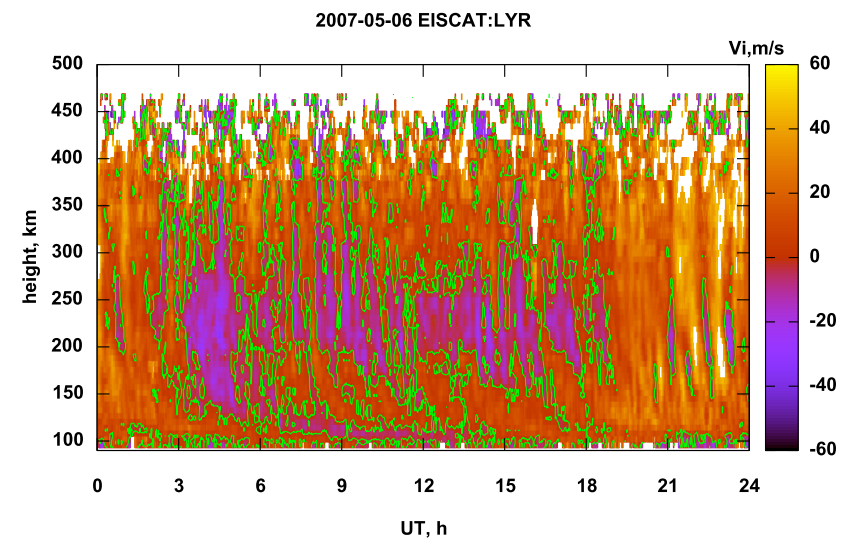

Fig. 3. The measured line-of-the-sight ion velocity data on 6 May 2007. Blank spots represent data points outside of the colorscale range.

Assuming that our time series consist of periodic signal(s) and white Gaussian noise (a reasonable assumption for AGW-TID studies) the statistical significance, e.g. the probability that our estimated power exceeds a threshold value of $z$ (statistical significance level) by chance, can be calculated as in (Scargle, 1982)

$p(P>z) \equiv 1-\left(1-e^{-z}\right)^{M}$,

so

$z=-\ln \left(1-(1-p)^{-M}\right)$

where $M$ is the number of independent frequencies (i.e. number of data points in the frequency domain), which is recommended to be at least 4N (Press and Teukolsky, 1988). Note that the significance levels depend only on the number of data points since the spectral power is normalized.

In order to apply spectral analysis, we interpolate the original data (which is initially unevenly sampled in altitude) to the uniform 64-point grid in altitude with height steps chosen so that the whole new grid is inside the original data domain. An example of interpolated ESR line-of-sight ion drift velocity data from 6 May 2007 is shown in Fig. 3. Another necessary preparatory step before the spectral analysis is to remove the "slow" daily trend. In this work we estimate the quadratic trend by doing a least-squares fit of a 2nd-order polynomial to the time series of each altitude level (for an example see Fig. 4 for 6 May 2007; altitude level is $228.5 \mathrm{~km}$ ).

The spectral analysis is applied on each altitude level independently (for an example, see Fig. 5). Then the dominant harmonics for each altitude are estimated, and harmonics present at an extended range of altitudes are detected. We have to use the Lomb-Scargle periodogram approach in an iterative manner, since the frequency spectra of the ISR data might contain many peaks : first the most powerful harmonic is estimated and removed, then the next most powerful is estimated and removed, and so on until the powers of the remaining harmonics are below the statistical significance level of

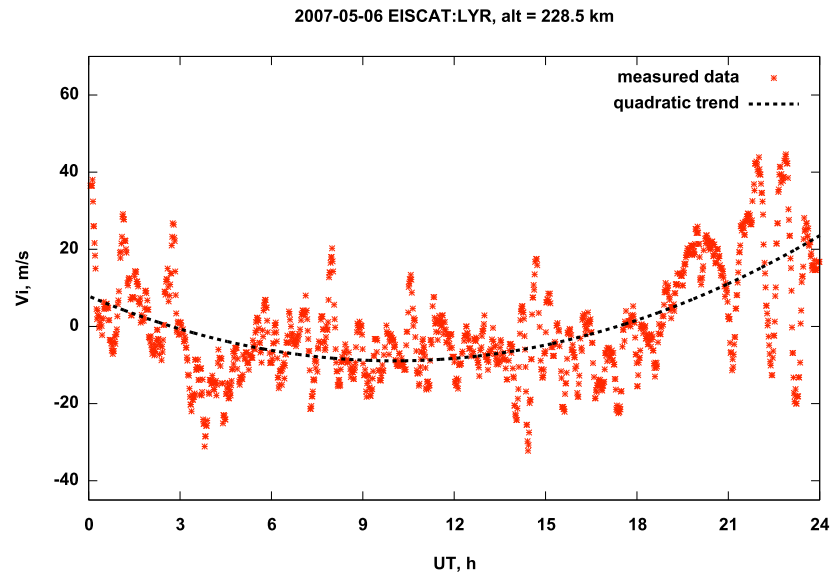

Fig. 4. Field-aligned ion velocity at the altitude $228.5 \mathrm{~km}$ on 6 May 2007 and the estimated quadratic trend.

$50 \%$. This means that the probability to observe the remaining harmonics just by chance is more than $50 \%$ (on each level independent of the others). This way we estimate the parameters of dominating harmonics for each altitude.

In the next step the distribution of periods of the harmonics is investigated for all the altitude levels combined, and the modal value(s) (most frequently observed) of this distribution is used as the central period for the pass-band of a filter. In order to get sharper distributions we have used weights inversely proportional to the order of the spectral power of harmonics, so that in each altitude level the dominant harmonic contributes 1.0 to the total count within the bin of the distribution histogram corresponding to the period of that harmonic, the second significant contributes 0.5 , the third one $0.33(3)$, etc. The width of the pass-band is $0.2 \mathrm{~h}$ (this value has been chosen based on filter tests). The period distribution for line-of-sight ion velocity, observed on 6 May 2007 is shown in Fig. 6 and one can easily see that one of the modal values of the distribution is around $1 \mathrm{~h}$. Our software gives for this case a band-pass filter with range of $0.92-1.12 \mathrm{~h}$. It should be noted that not only the most often observed value is used in further analysis as a filter pass band but also up to 10 other modal values of period distribution.

This approach allows us to avoid an analysis of measurement error statistics, as long as we assume that the measurement errors are random noise (its autocorrelation function is a delta-function), and our aim is search and extraction of periodic signals. The input data is treated as a sum of periodic signal(s) (harmonics) and random noise, as this is assumed by the Lomb-Scargle spectral analysis. The results of the spectral analysis show both how close the input data is to this model and how well we can define the periodic components. Furthermore, after the spectral analysis applied to the data from each altitude independently, this step combines the results in order to find whether any periodicities are detected on a number of levels simultaneously. Like described 


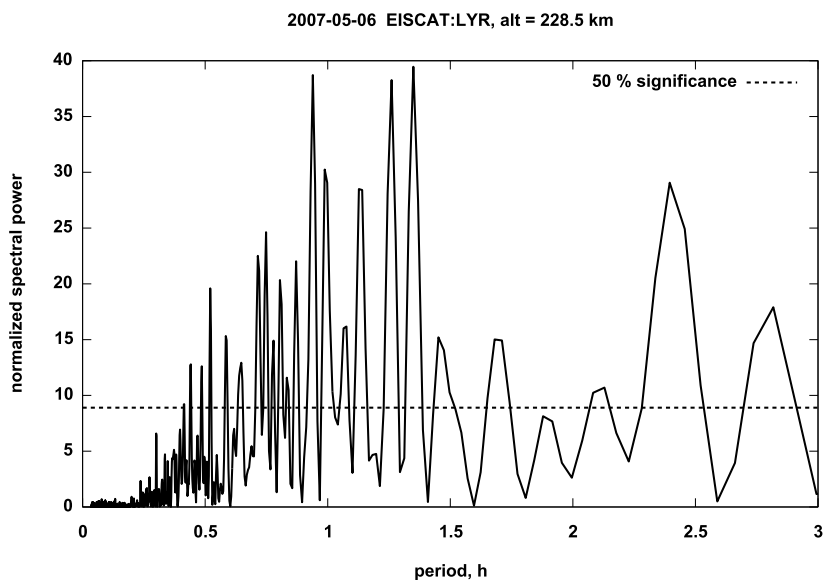

Fig. 5. Lomb-Scargle periodogram of the field-aligned ion velocity at the altitude $228.5 \mathrm{~km}$ on 6 May 2007 and the $50 \%$ statistical significance level (horizontal dashed line).

above, for each altitude we only consider estimates that are above $50 \%$ statistical significance level. In other words, the probability that the detected harmonic component occurred just by chance is $50 \%$ or smaller. When results from a number of altitudes are combined, the resulting statistical significance is obtained via probability multiplication, i.e. for the same period identified on two altitude levels simultaneously the probability of occuring by chance is at most $25 \%$, in the case of 3 altitude levels $12.5 \%$ and it is below $1 \%$ in the case of more than 6 levels showing the same harmonic component. This way the power of the signal (the one we are looking for) in the frequency band, determined by our analysis, is much higher than the power of noise. If we then apply the filter with the corresponding pass-band, the output would be mainly defined by a real physical wave and not by random noise.

In our case the only remarkable disadvantage of use of the Lomb-Scargle periodogram may be, that the frequency of AGW-TID in the real data is not constant. This leads to a flattening of spectral peaks (they become lower and broader, since more spectral power goes to the neighbour frequencies). Nevertheless any statistically significant periodicity will be successfully detected, although the estimated passband for a filter might be not optimal. One of the ways to overcome this difficulty is to use a filter whose slopes at the cut-off frequencies are not too steep.

\subsection{Filtering}

Similarly as in previous TID studies (Hocke and Schlegel, 1996) we use Butterworth filters, because they have a smooth response function and are computationally efficient, being recursive filters. Butterworth filters, however, have two drawbacks: (i) they generate a phase shift between input and output, and (ii) there are significant distortions at the beginning of the output record. The first problem is solved by applying

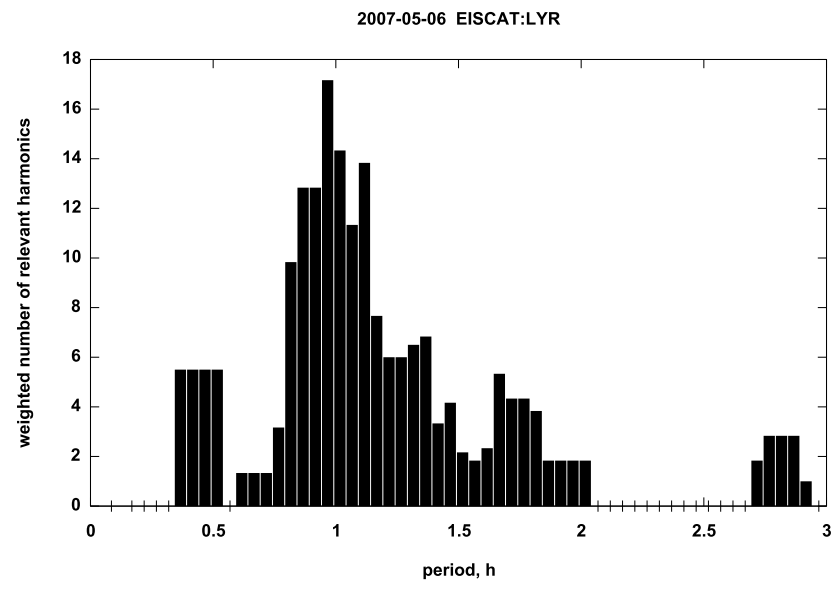

Fig. 6. Histogram of the dominant periods on 6 May 2007 (all altitude levels and time steps combined).

the same filter twice: first in forward direction and then in backward direction. The second problem is solved by introducing relaxation zones at both edges of the data record. The relaxation zones are constructed using the relevant harmonics determined during the spectral analysis step (see previous chapter). A Hamming window is applied to the data inside the relaxation zones in order to provide a smooth decay towards zero at the outer edges of both relaxation zones.

Before filtering we need to do two prepatory modifications in the data: fill the gaps (with the help of the output from the Lomb-Scargle periodogram, see discussion above) and do some downsampling in order to reduce the length of the time series. The downsampling is done by $2 \mathrm{~min}$ averaging. After applying the filter, the artificially created data points inside the gaps and the relaxation zones are removed.

The filter itself consists of two second-order bandpass Butterworth filters (one applied forward and one backward). The reason for such low order is, that the flat response function is desired (see previous section). The transfer function is (Hamming, 1977)

$H\left(T, T_{1}, T_{2}\right)=\sqrt{\left(\frac{1}{1+\left(\frac{W(T)}{W\left(T_{1}\right)}\right)^{4}}\right)^{2}\left(1-\sqrt{\left.\frac{1}{1+\left(\frac{W(T)}{W\left(T_{2}\right)}\right)^{4}}\right)^{2}}\right.}$

where $T$ is period, $T_{1}$ and $T_{2}$ are band-pass boundary periods $\left(T_{2}>T_{1}\right), W(T)=\tan \left(\pi \frac{T_{\mathrm{S}}}{T}\right), T_{\mathrm{S}}$ is time step.

The filter significantly suppresses signal even within the bandwidth, so in order to properly estimate the amplitude, we have to use a correction factor

$K=\frac{1}{H\left(\bar{T}, T_{1}, T_{2}\right)}$

where $\bar{T}=\left(T_{1}+T_{2}\right) / 2$. 


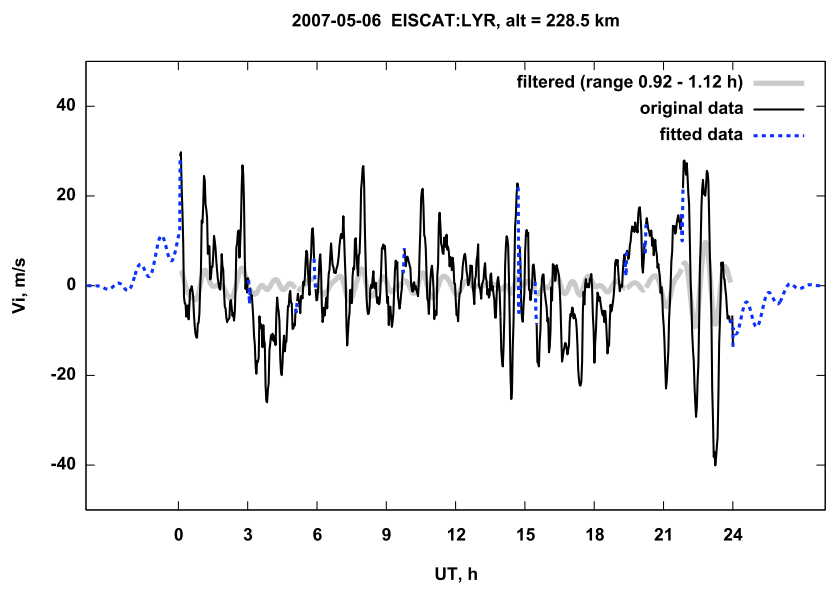

Fig. 7. Detrended line-of-sight ion velocity data from altitude $228.5 \mathrm{~km}$ for 6 May 2007 with filled gaps and added relaxation zones and the output of filter with band-pass centred around the dominant harmonic.

The output of the filter is then interpreted visually using criteria formulated in Sect. 2.2, and ambiguous data samples are removed from further consideration. Figure 7 shows the ion drift velocity data from altitude $228.5 \mathrm{~km}$ for our example case with the relaxation zones added and gaps filled, as well as the output of the filter with the band-pass centered at the most often observed period of the analysed day (gray thick line). In the filtered altitude-versus-time plot for the entire day (Fig. 8) one can easily see the quasi-periodic disturbances with "downward" phase progression in time as typical AGW-TID signatures.

\section{Results and discussion}

\subsection{TID periods}

The whole ESR IPY-dataset was analyzed in the way described above in portions equal to amount of data available within one UT calendar day. We assumed that multiple AGW-TIDs with different periods may be observed simultaneously and they are treated as separate cases of AGW-TID in our statistical analysis. The distribution of periods as observed in all the four ISR parameters during the IPY run are shown in Fig. 9. The distributions tend to have a two-peak structure and one can easily distinguish the bands of the most common periods (modes) for each parameter: $0.7-0.9 \mathrm{~h}$ and $1.1-1.3 \mathrm{~h}$ in electron density variations and in ion and electron temperature variations. In the line-of-sight ion velocities the maxima are at $0.5-0.7 \mathrm{~h}$ and $1.1-1.3 \mathrm{~h}$. It is worth notifying that the ion velocity statistics is of greater importance because of the significantly larger number of cases. If we compare our periods with the previously mentioned categorization for Medium-Scale TIDs (periods less than an hour) and Large-scale TIDs (periods $>30 \mathrm{~min}$ ) we notice that most

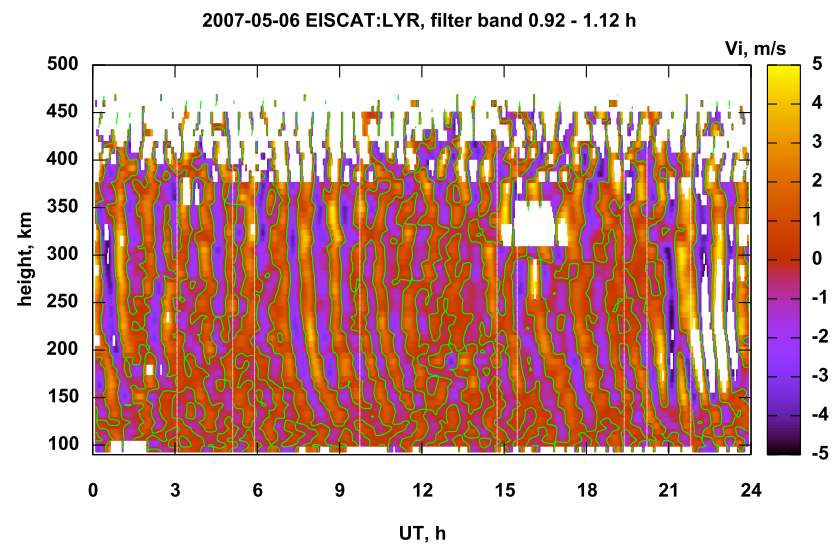

Fig. 8. Filtered field-aligned ion velocity data on 6 May 2007.

of the events in our data base belong to the "gray" area where both Large- and Medium-Scale TIDs can exist (keeping in mind, though, that the oscillation period is not the only factor discriminating these two wave types). The highest normalized occurence rate (exceeding 0.5, i.e. more than $50 \%$ of the cases) is in the histogram of electron density and it is at the periods of $0.4-0.8 \mathrm{~h}$, which are typical for MSTIDs.

\subsection{Seasonal and diurnal AGW-TID occurrence rates}

The normalized monthly occurrence frequency of the AGWTID events in all the observed quantities $\left(N_{\mathrm{e}}, T_{\mathrm{i}}, T_{\mathrm{e}}, V_{\mathrm{i}}\right)$ is displayed in Fig. 10. In our dataset TID occurrence has its maximum during summer months (May-July) and minimum during winter months (November-January). In order to minimize the possible influence of the variations in the data coverage we normalized the monthly occurrence frequency by the number of days with observations during the analysed month (cf. Fig. 1). The winter months during the IPY run are much less covered by observations than the summer months. The poor data coverage might have an impact on the seasonal AGW-TID occurrence rate. Another possible explanation behind the observed annual cycle might be seasonal differences in the ionospheric electron density, which defines the ISR backscatter intensity and hence signal-to-noice ratio. One can see a good correlation between Total (integrated over line-of-sight) Electron Content measured by ESR during IPY (Fig. 11) and the monthly occurrence frequency of AGW-TID.

The obtained annual distribution of AGW-TID occurrence is in good agreement with the results of Kotake et al. (2007) (for nighttime MSTIDs) but at the same time it is in contradiction with AGW-TID climatology reported for SuperDARN (Bristow et al., 1996), which shows a maximum of AGW activity around wintertime and a minimum around summertime. The AGW-TID detection method in our study and that of Bristow et al. (1996) differ from each other in many respects. First of all, the radar field-of-views 


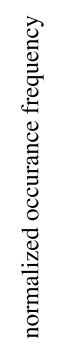
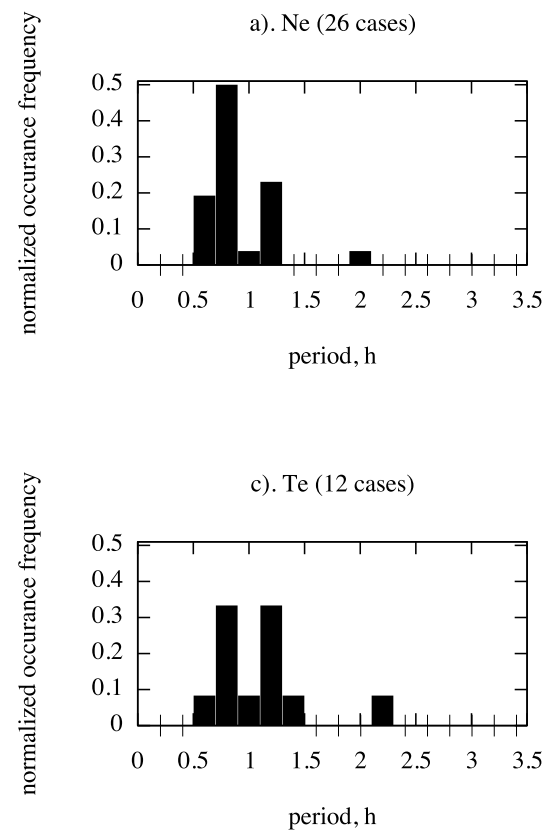
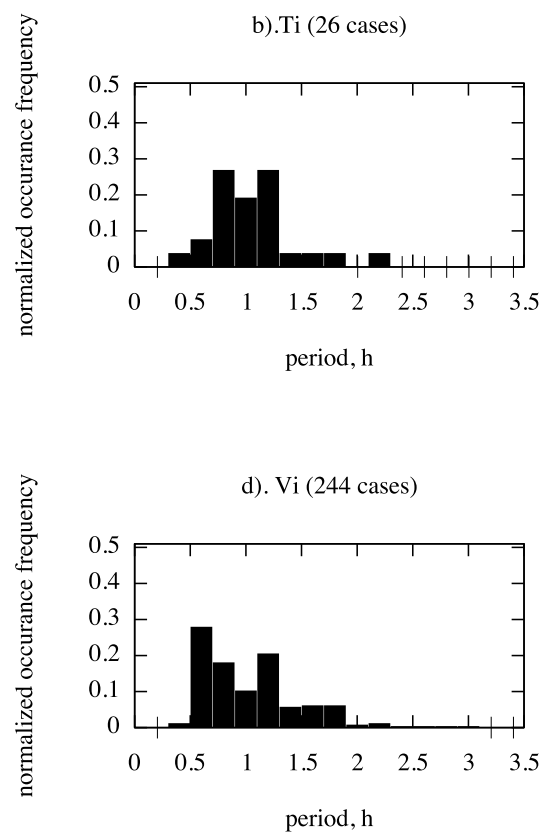

Fig. 9. Histogram of the oscillation period for (a) electron density, (b) ion temperature, (c) electron temperature, and (d) field-aligned ion velocity. For each parameter the occurrence rates have been normalized with the total number of AGW-TID cases observed in that parameter.

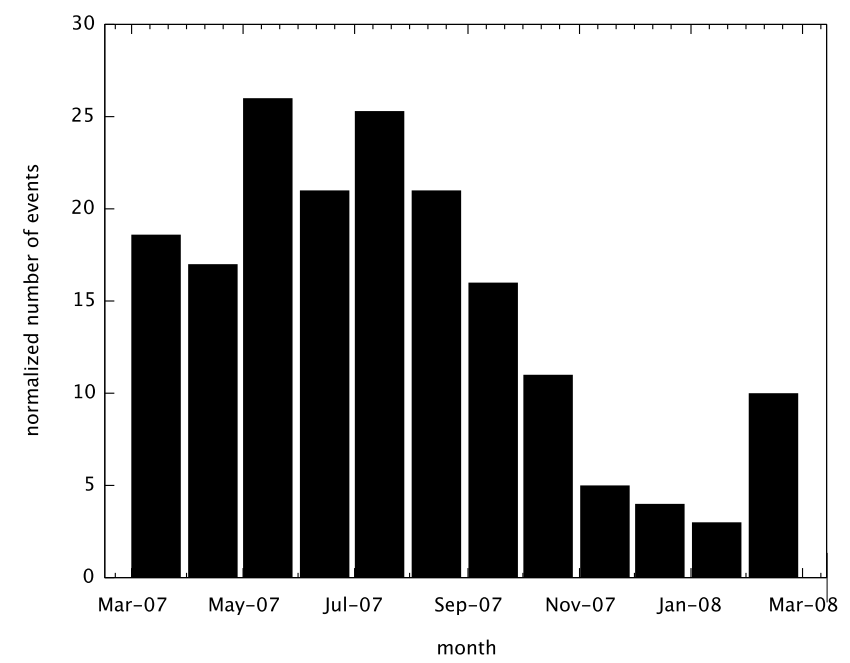

Fig. 10. Normalized monthly occurrence frequency of AGW-TID $\left(N_{\mathrm{e}}, T_{\mathrm{i}}, T_{\mathrm{e}}\right.$, and $V_{\mathrm{i}}$ combined) signatures in ESR IPY data. The height of the bars tell the number of days with AGW-TID-signatures observed in each month, divided by the ratio of the number of days with ESR data to the total number of days within the same month.

are different: SuperDARN collects backscatter from a twodimensional horizontal area with typical scales of some hundreds of $\mathrm{km}$, while the ESR antenna collects backscatter only along one narrow (1-3 km wide) beam, but at a number of altitudes along the field line. SuperDARN radars observe AGW-TIDs from ground-scatter data and they provide information also about the horizontal wavelengths for event detection. This method, however, does not provide any infor- mation about the vertical phase distribution, which is one of the principal criteria for AGW-TID in our analysis. Furthermore, the SuperDARN method used by Bristow et al. (1996) detects mainly MSTIDs while our dataset may contain also LSTIDs. The fact that the ESR-IPY data have been collected in exceptionally deep solar minimum conditions might be another reason for the difference of our AGW-TID occurrence from the previous observations. In particular, the deep minimun in the winter time in our histograms is likely to be a consequence of the low background electron content, caused by the combined effect of low solar zenith angle and dramatically decreased level of solar activity.

We also inspected seasonal variations in the AGW-TID periods for four calendar seasons: spring (March, April, May), summer (June, July, August), fall (September, October, November) and winter (December, January, February), as shown in Fig. 12. Only cases, when AGW-TID were observed in ion velocity have been considered because of the much smaller amount of AGW-TID cases for other measured quantities. From Fig. 12 we can conclude that the most often observed periods of AGW-TID are the same all the year through. The histograms of spring and summer have a similar two-peak structure as the histogram of all ion velocity events (Fig. 9). Winter and fall occurrence rates of AGWTID periods should be considered with caution because of the much smaller number of observed AGW-TID cases compared to other seasons. One can see, however, that these histograms have sharp maxima of occurrence rate around the period of $0.6 \mathrm{~h}$, which suggests that MSTIDs are more frequent in these datasets. 


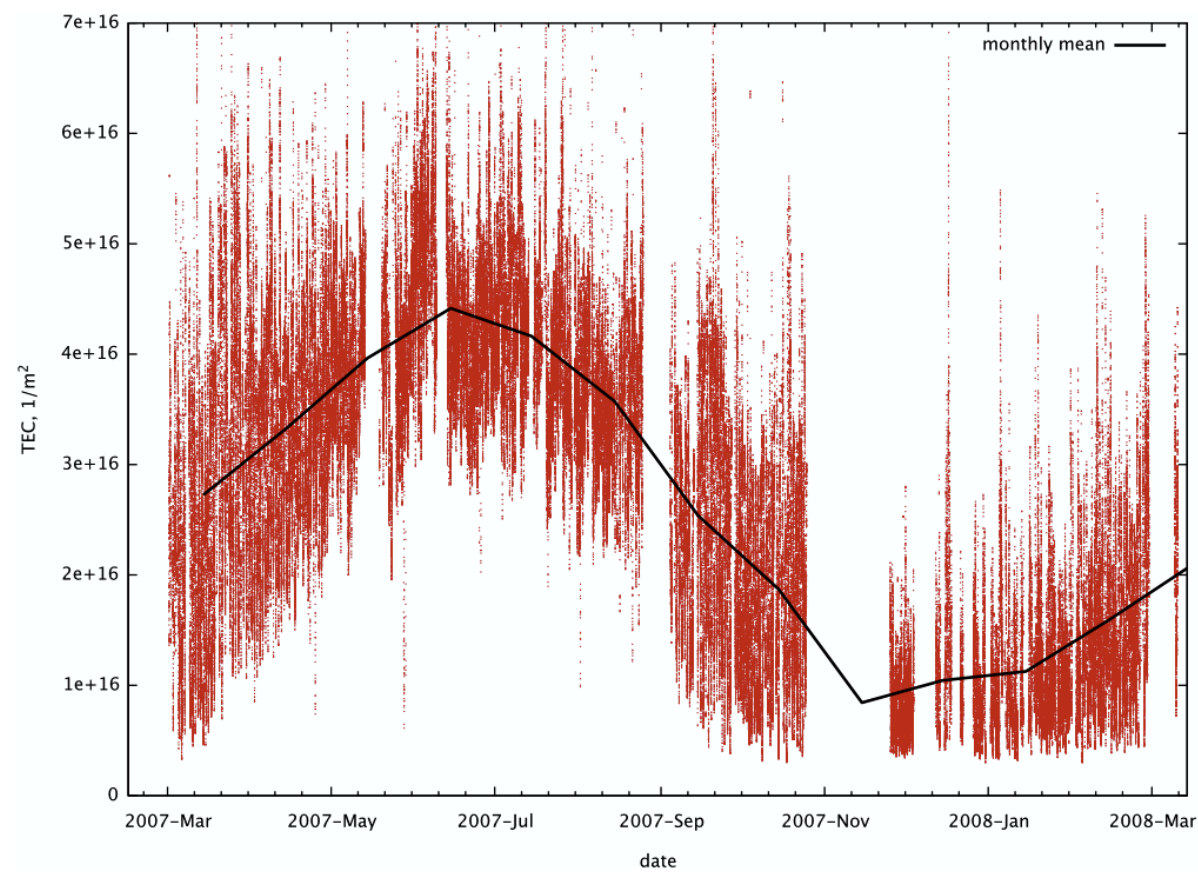

Fig. 11. Total Electron Content observed by ESR during the IPY-run (individual measurements and monthly mean).
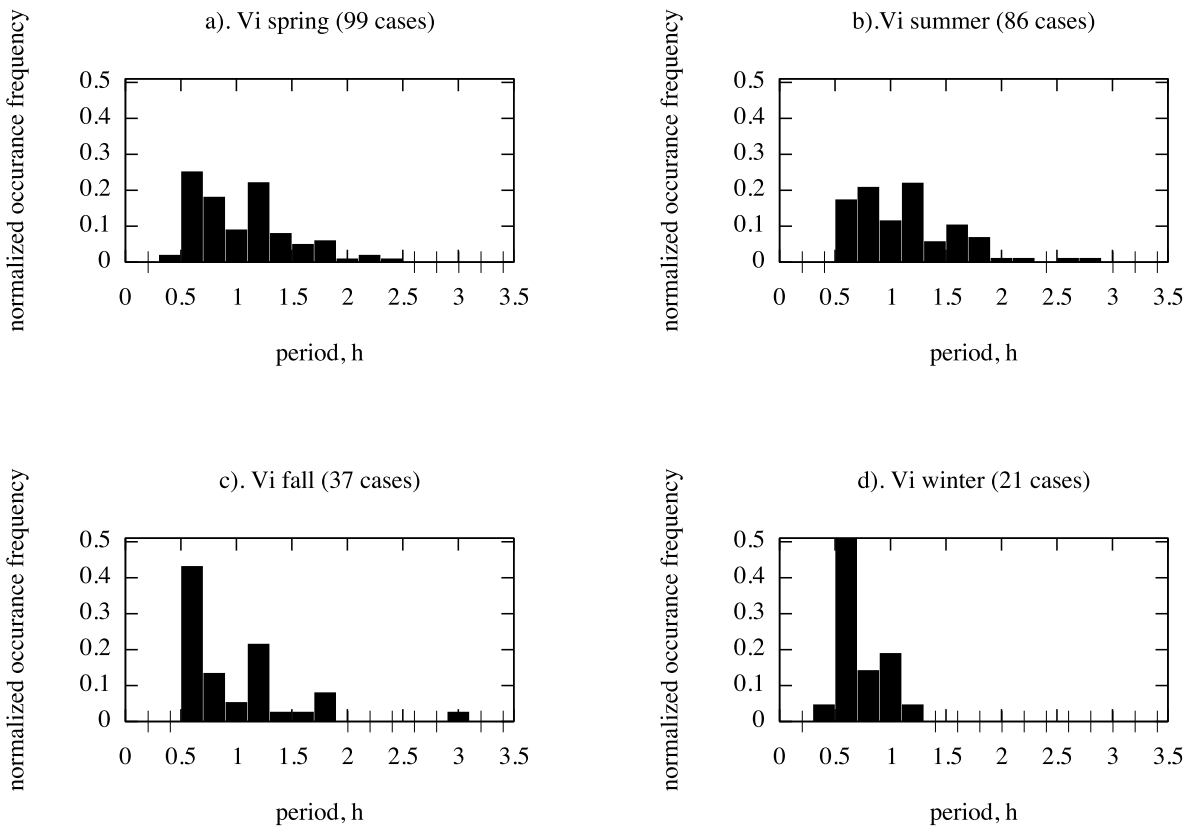

Fig. 12. Seasonal dependencies in the histograms of AGW-TID periods as observed in the ion velocity data.

The diurnal variability of the AGW-TID occurrence frequency for the whole data-set is presented in Fig. 13. We have divided the day into four-hour blocks and for each block counted the cases when TID signatures existed in that particular block. The occurrence frequency is the number of events when AGW-TID have been observed within the given 4-h time block divided by the overall number of cases (declared in parenthesis). It is clearly seen that hardly any AGW-TID events were observed between 20:00 and 24:00 UT. As in Svalbard the magnetic local time is roughly two hours ahead of the UT-time, this slot corresponds to the midnight sector ( 22:00-02:00 MLT), and the Harang discontinuity region (Pryse et al., 1996) where auroral substorm activity is often stronger than elsewhere, which thus may obscure the TID 
a). $\mathrm{Ne}(26$ cases $)$

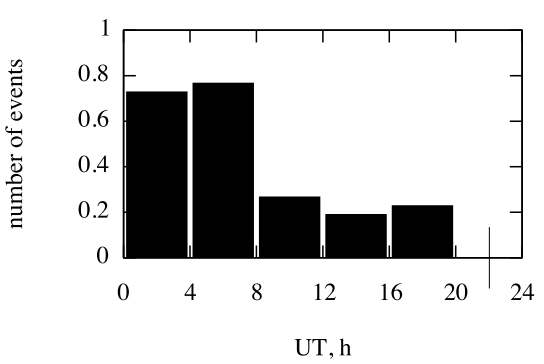

c). Te (12 cases)

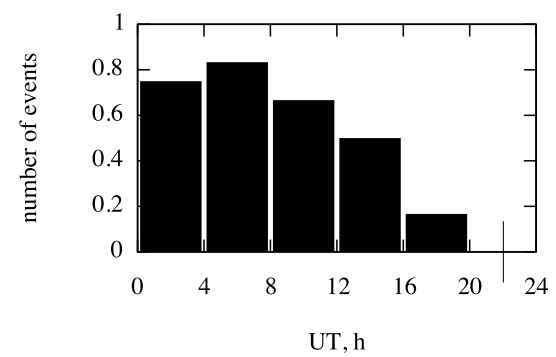

b).Ti (26 cases)

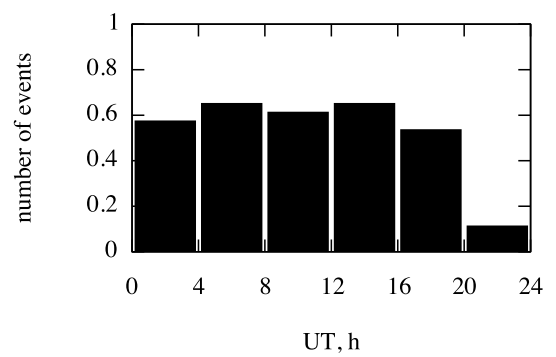

d). Vi (244 cases)

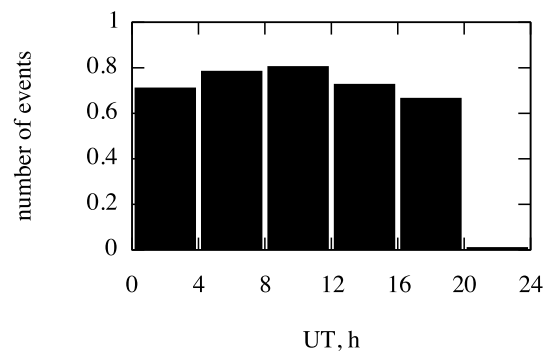

Fig. 13. AGW-TID occurrence rate as a function of UT for the all the four ISR parameters.

signatures. Outside the midnight sector the distribution of occurrence frequency (especially in ion velocity) is smooth and almost uniform with slight maxima around morning and noon hours. The distribution in $N_{\mathrm{e}}$ shows a notable preference for morning hours but as the total number of the observed events is quite low we are reluctant to draw any conclusions from this finding.

We studied also the diurnal occurrence rates during the different seasons. We used only data for ion velocity cases because of the very limited amount of AGW-TID cases for other variables. We divided the ion velocity dataset to four bins according to calendar seasons (the statistics are shown in Fig. 14). The results for spring and summer look quite similar to the overall picture (Fig. 13). In the histograms of fall and winter a tendency of lower occurrence rates in the evening and night is visible. A likely explanation for this trend is that detecting AGW-TIDs in the dilute dark winter ionosphere with ISR is more challenging than during other conditions. Apparently it can't be caused by systematic gaps in the observations, since no regular diurnal variations in the ESR data coverage is present. Our diurnal distribution of AGW-TID occurrence is different from that observed using GPS measurements at mid-latitudes where night-time MSTIDs are observed preferentially in summer (Shiokawa et al., 2004) and daytime MSTIDs in winter (HernandesPajares et al., 2006). These differences may be explained by the different observing region: ESR is likely to be more often close to the source region than mid-latitude instrumentation (Ogawa et al., 2009). Consequently, the diurnal and seasonal distributions of mid-latitude MSTIDs are more affected by the wave propagation conditions in the atmosphere than the events in our dataset.

One of the most important parameters for analysis of AGW-TID cases is the horizontal phase velocity (Hunsucker, 1987; Kersley and Hughes, 1989), since it may allow to link observed ionospheric irregularities with particular generation processes or at least distinguish between lower and upper atmospheric sources. Such separation is of great importance for understanding of the AGW role in the atmosphereionosphere-magnetosphere system. Unfortunately in our case ESR provides only measurements of nearly vertical profiles at a single point. So the only way to estimate the horizontal scale of AGW is using one of the simplified theoretical dispersion relations. In our work we used the classical AGW dispersion relation derived by Hines (Hines, 1960):

$\omega^{4}-\omega^{2} C^{2}\left(k_{\mathrm{x}}^{2}+k_{\mathrm{z}}^{2}\right)+(\gamma-1) g^{2} k^{2}-\gamma^{2} g^{2} \omega^{2} / 4 C^{2}=0$

where $\omega$ is the angular frequency, $C$ is the speed of sound (assumed to be $=580 \mathrm{~m} \mathrm{~s}^{-1}$ in the thermosphere), $\gamma=1.51$ is the specific heat ratio, $g=9.3 \mathrm{~m} \mathrm{~s}^{-1}$ is gravity acceleration, $k_{\mathrm{x}}$ and $k_{\mathrm{z}}$ are horizontal and vertical wavenumbers, respectively. This dispersion relation assumes the background atmosphere to be horizontally uniform and isothermal (hence $C=$ const), with density stratification, and AGW to be a monochromatic wave. We also had to assume that the intrinsic (i.e. relative to the moving neutral atmosphere) frequency of AGW is equal to the observed one, since no reliable neutral wind data was available. The vertical wavelengths have been estimated visually with approximately $25 \mathrm{~km}$ accuracy after filtering as described in Sect. 2. The estimations of all 
a). Vi spring (99 cases)

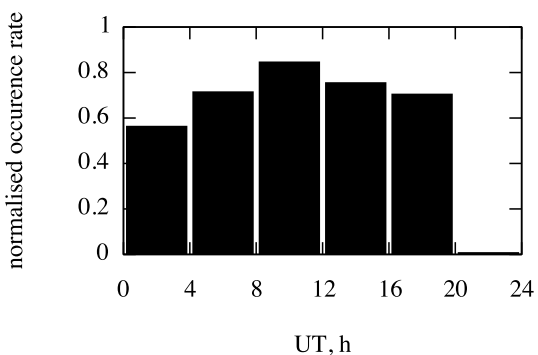

c). Vi fall (37 cases)

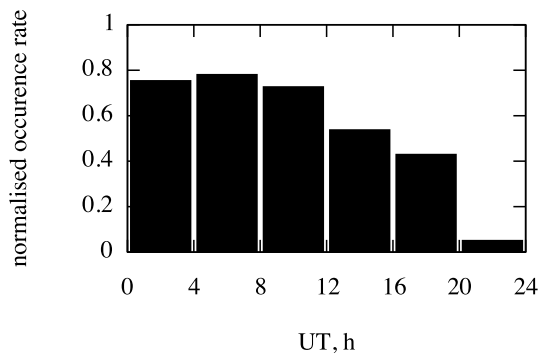

b).Vi summer ( 87 cases)

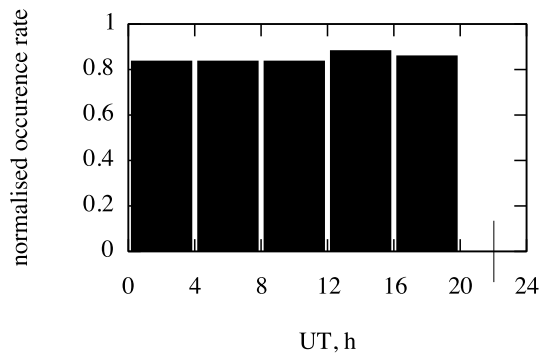

d). Vi winter (21 cases)

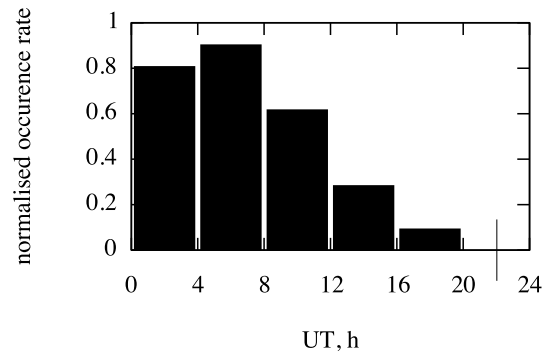

Fig. 14. AGW-TID occurrence rate as a function of UT for different seasons.

the cases are presented in Fig. 15 as a function of period. Based on simulations presented in Kirchengast (1996) and Kirchengast (1997) vertical wavelengths of AGW might be assumed to be roughly equal in all ISR parameters, except for $T_{\mathrm{e}}$ (Kirchengast, 1997), which wasn't used for the estimation of horizontal wavelengths. The overall tendency in our result is well pronounced: as expected, with increasing periods we get larger horizontal wavelengths. Another remarkable feature is that the datapoints from the $N_{\mathrm{e}}, T_{\mathrm{i}}$ and $V_{\mathrm{i}}$ events are inside the same "cloud". This suggests that all the observed AGW-TID events are of the same type and most probably the same origin as well. The average horizontal phase speed (wavelength/period) is around $250 \mathrm{~m} \mathrm{~s}^{-1}$, so the observed AGW can be classified as those on the "border" between large-scale and medium-scale AGW (Kersley and Hughes, 1989). This result, however, should be confirmed in future work where the vertical wavelengths are estimated more accurately and the effect of neutral wind on this wavelength is taken into account.

\subsection{AGW-TID amplitudes}

In order to draw any conclusions about the role of AGW-TID in the dynamics of the neutral atmosphere and ionosphere it is necessary to have information about their amplitudes. Here we study only the seasonal variations in the amplitudes. A detailed analysis of temporal variations in AGW amplitudes and the relation of the amplitudes with geomagnetic activity and the neutral atmosphere dynamics is left for future studies.

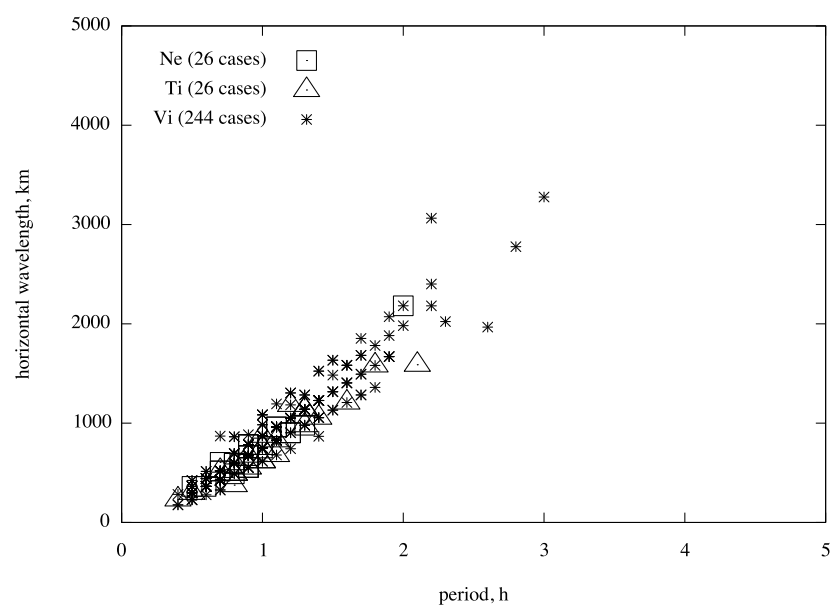

Fig. 15. Dependency of the estimated horizontal wavelength on the oscillation period as based on the AGW dispersion relation by Hines (1960).

For getting the amplitude estimates we use the following procedure in order to reduce possible errors caused by the fact that observed AGW-TIDs are quasi-periodic (may have non-constant periods). We study the data that have been processed according to the flow chart shown in Fig. 2 and the visual inspection steps after it. In the analysis we use similar four-hour bins with AGW-TID activity as used in the diurnal occurrence analysis. For each block and for each altitude the AGW-TID period is known from our previous analysis. Then we use a sliding window with length in time equal to 

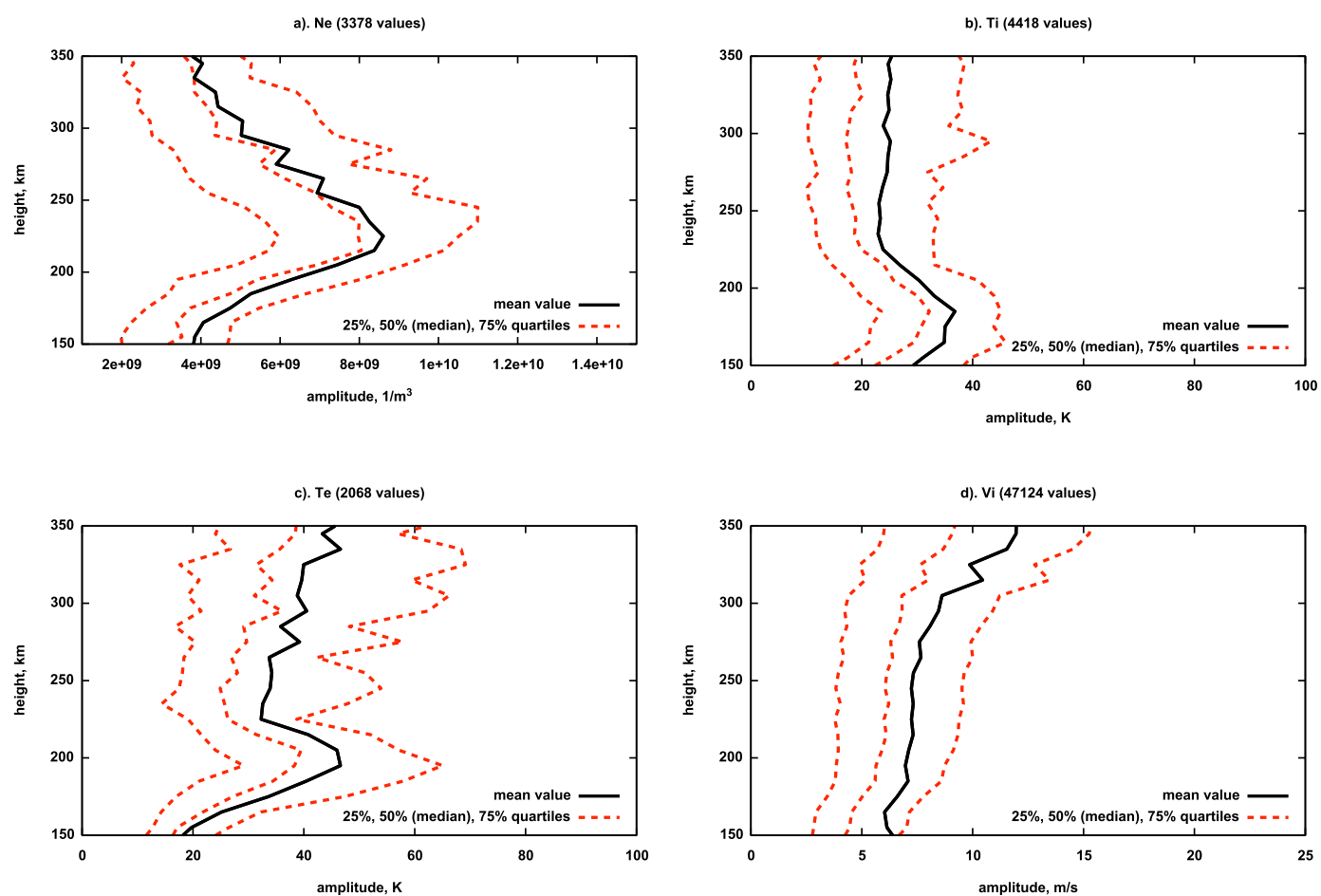

Fig. 16. Amplitude variations as function of altitude as observed in all the four ISR parameters. The black curve shows the average. The middle half of observed amplitudes is within the leftmost and rightmost red dashed lines (the dashed line in the middle is the median value). The number of datapoints given in the titles of the plots is the sum of points from all altitude levels.

the known period. At each step for the data inside the window, we do a least-square fit with a harmonic wave of the dominating period in order to get the wave amplitude and phase. The obtained amplitude values have been corrected using Eq. (6). In the next steps of the analysis we use the average values (over four-hour bins) of these corrected samples. The analysis has been done for the whole IPY-period and for each variable. For $V_{\mathrm{i}}$ we have done binning to four calendar seasons, since it is the only variable with sufficient amount of AGW-TID events. The amplitude analysis has been performed for the altitude range $150-350 \mathrm{~km}$ for both the whole year dataset and for the $V_{\mathrm{i}}$ seasonal datasets, since the AGW-TIDs have not often been clearly seen outside these domains.

The results are summarised in Fig. 16 and Fig. 17. In addition to averaging we also calculated median values together with $25 \%$ and $75 \%$ quartiles, like it was done by Hocke et al. (1996). It can be seen from the plots that the mean values are close to the median values, but slightly higher (probably due to asymmetry of the distribution). In general our results are similar to those obtained by previous researchers. $V_{\mathrm{i}}$ amplitudes obtained in our study are slightly higher than presented earlier in the literature $\left(5-7 \mathrm{~m} \mathrm{~s}^{-1}\right.$ at F-layer heights in our case compared to $2-6 \mathrm{~m} \mathrm{~s}^{-1}$ reported by Hocke et al. (1996)). Our seasonal analysis shows that the altitude profile of AGWTID amplitudes in ion velocity looks quite the same all the year through except during winter when the amplitudes on all the levels are higher. This tendency needs further confirmation in future studies, however, since the number of events in the winter bin is much smaller than in the other bins.

\subsection{Geomagnetic activity}

In many previous studies (see, for example, a review by Hocke and Schlegel, 1996) variations in electric currents and particle precipitation events have been suggested as possible sources of medium-scale AGW at high latitudes. In order to investigate relations between geomagnetic activity and observed AGW-TID we decided to use IE-index (IMAGE electrojet index) (Kauristie et al., 1996) derived from observations on IMAGE regional magnetometer network. The IMAGE network covers Scandinavia and the Svalbard archipelago, so these data are affected only by processes in this area, unlike global indices, e.g. AE-index. By definition, a sudden increase in IE-index is caused by energy load from the magnetosphere and intensification of the ionospheric currents. Figure 18 compares 1 min IE-index data with the observed AGW-TID occurrence frequency (cf. Fig. 10). One can see that the IE-index behaviour is more or less uniform throughout the IPY-run and does not show similar seasonal variation as the AGW-TID occurrence rates. At the beginning of 2008 the IE-activity variance increased somewhat while the AGW-TID activity decreased, but this is likely a pure coincidence since the two datasets do not 

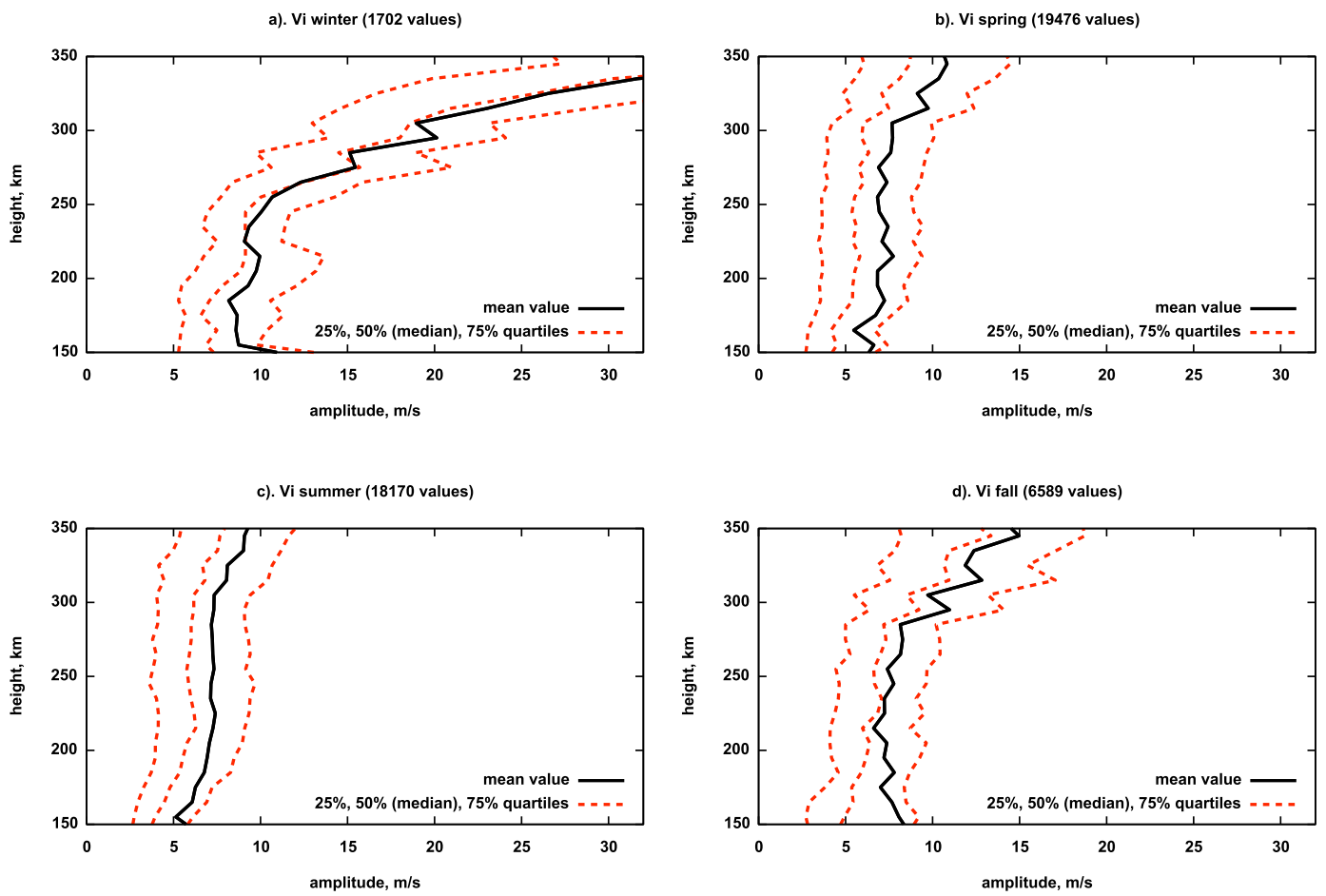

Fig. 17. Amplitude variations as function of altitude as observed in ion velocity data during the different seasons. The black curve shows the average. The middle half of observed amplitudes is within the leftmost and rightmost red dashed lines (the dashed line in the middle is the median value). The number of datapoints given in the titles of the plots is the sum of points from all altitude levels.

otherwise show any correlation. In Fig. 19 the distribution of IE-index during the whole IPY-run is compared with the distribution of IE-index observed during AGW-TID events. The distribution of all IE-index points shows two maxima, one around $3 \mathrm{nT}$ and the other around $13 \mathrm{nT}$. As IMAGE stations are not permanent magnetic observatories their measurements contain variable baselines, which have to be removed. The errors in baseline removal most probably cause the peak around $3 \mathrm{nT}$, while the latter peak is more likely to represent the true most frequent activity level. The distribution of the IE values recorded during the AGW-TID events is unimodal with the occurrence frequency peak at $\sim 18 \mathrm{nT}$. This peak is at $\sim 5 \mathrm{nT}$ higher than the peak in the IE distribution of the whole IPY-period, but on the right side of the peak point the two distributions are very similar, which leads us to believe that increased geomagnetic activity was not the main driver of the AGW-TID events in our dataset. The fact that the seasonal variability of AGW-TID occurrence rate doesn't have peaks during equinoxes in Fig. 10 (contrary to Tsugawa et al., 2004) further confirms that the IPYrun AGW-TIDs most probably were not LSTIDs.

\section{Summary and conclusions}

The goal of this paper is to report the statistics of AGW-TID observations from a new unique ISR dataset: the one year
IPY-run by the EISCAT Svalbard Radar. TIDs in our data base have features which according to previous studies show their association with Atmospheric Gravity Waves (Hines, 1960). All of our events show the downward propagating phase fronts that are typical in ISR data for AGW-TIDs. Below is a short summary of our main results:

1. It has been shown that AGW-induced TID's are very common above Svalbard. Although expected, this is the first time it has been confirmed. The ESR AGW-TID observations are in a good agreement with previously reported cases from other Incoherent Scatter Radars, including those from Troms $\varnothing$ (Kirchengast et al., 1995) and Arecibo (Djuth et al., 1997).

2. AGW-TIDs have been observed at Svalbard more often during spring and summer than during winter and fall months. Confirming this tendency would need a longer dataset and it is important to note that the IPY run was conducted during deep solar minimum conditions. The seasonal distribution resulting from this study is different from the results of previous studies, where LSTIDs seem to follow the Russell-McPherron distribution (peaks at equinoxes) (Tsugawa et al., 2004) and the MSTID occurence has maxima both in summmer and winter (Kotake et al., 2007). The seasonal variation in AGW-TID occurrence achieved in this study follows 


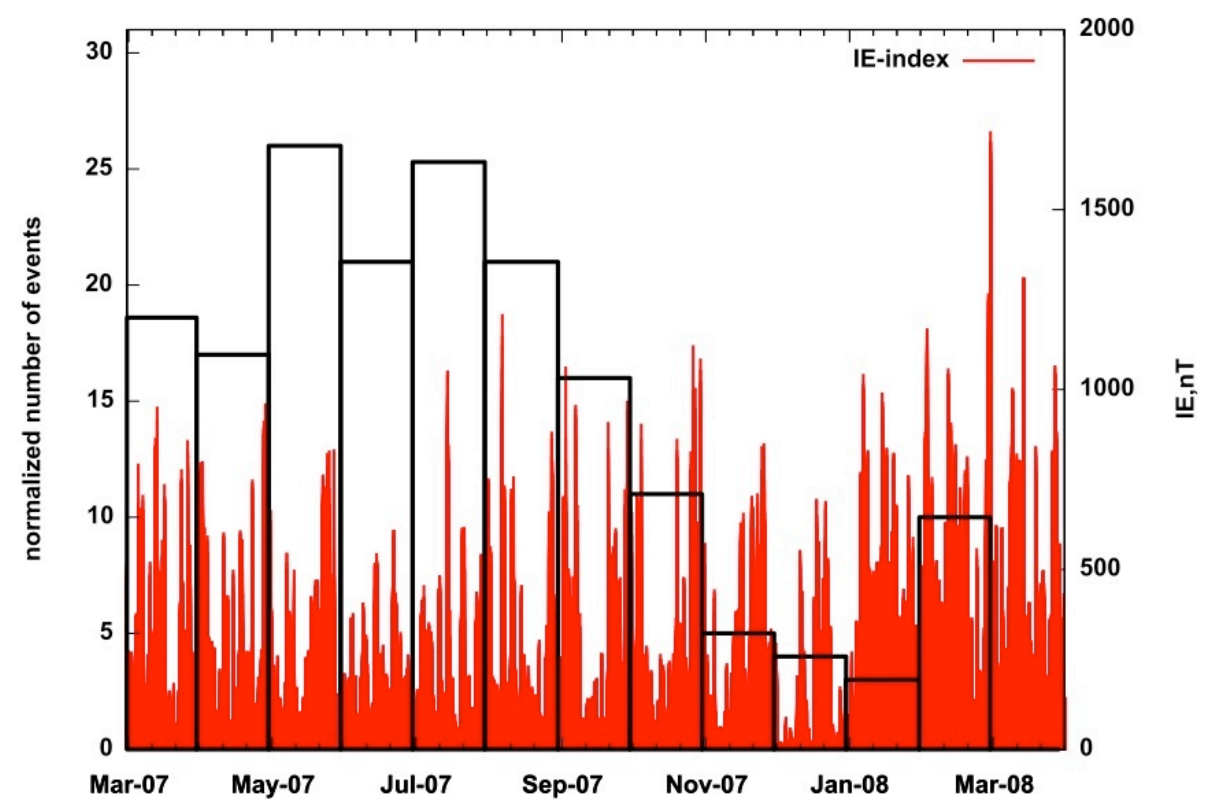

Fig. 18. The IE-index during the IPY-run and the normalised AGW-TID occurrence frequency (black bars; same data as in Fig. 10).

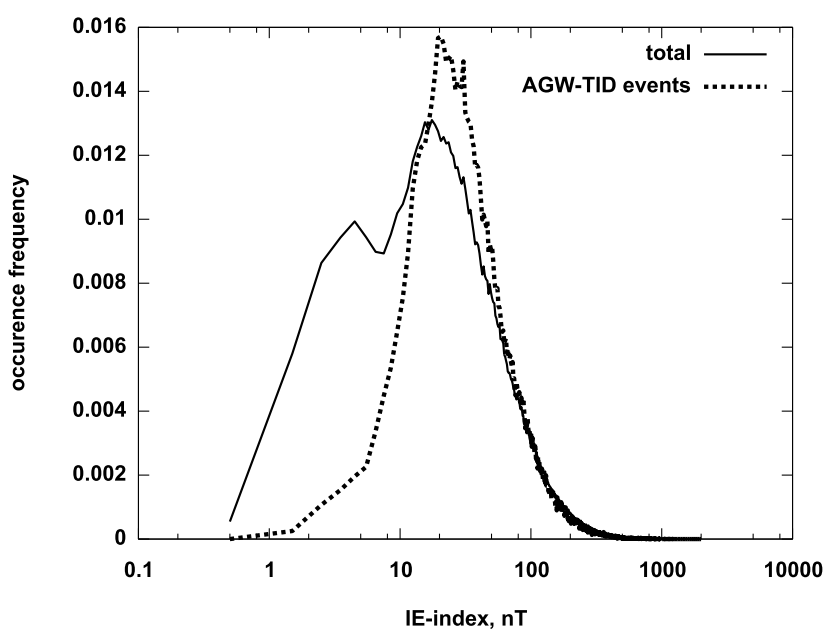

Fig. 19. The distributions of IE-index values recorded during the whole IPY-run (solid line) and of those recorded during the AGWTID periods. The distributions have been normalized with the corresponding total numbers of IE-values.

closely the seasonal variation of ionospheric electron content at the observing site. It remains still as a topic for future studies whether the observed seasonal variation is natural or caused by seasonal variation in the performance of our observation method.

3. The most often observed periods in AGW-TIDs are in the bands $0.5-0.7 \mathrm{~h}$ and $1.1-1.3 \mathrm{~h}$. These results are consistent with previous findings about the most common AGW-TID periods. If we compare our periods with the previously mentioned categorization for
Medium scale TIDs (periods less than an hour) and Large scale TIDs (periods $>30 \mathrm{~min}$ ) we notice that most of the events in our data base belong to the overlapping area where both Large and Medium scale TIDs can exist.

4. The AGW signatures are clearest in ion velocity data. One has to remember that if we don't see the signatures in the other ISR parameters it doesn't mean their total absence. For example, in electron density the AGW signatures can be masked by some other background processes. On the other hand, we can conclude that at latitudes of Svalbard AGW-TIDs should be of minor impact for the radio wave propagation applications (e.g. HF-communication or satellite navigation), for which usually only the electron concentration distribution matters.

5. The obtained statistics of AGW-TID amplitudes are quite consistent with results from previous studies. No remarkable seasonal variation of Vi-AGW-TID amplitudes have been found.

6. Inside the day AGW-TIDs are equally commonly seen at all hours except the time interval close to midnight UT. The drop in the midnight sector in our data set is most probably caused by masking of AGW-TID activity by other processes in the highly dynamic auroral ionosphere.

7. The estimated horizontal scales of AGW-TID do not allow to draw any conclusions about the sources of those waves. The intercomparison with other measurements is still required. 
A subsequent analysis of the ESR-IPY dataset including detailed case studies, a detailed statistical study of amplitudes, comparison with other instruments and theoretical modelling will provide a deeper insight into the physics of AGWs and their role in coupling between different geophysical processes at the poleward edge of the auroral oval. Having a three-dimensional view of the ionospheric conditions would be of significant help in this work. At auroral latitudes such studies have already been conducted with the multi-beam capability of the new generation phased-array ISR at Poker Flat Research Range (PFISR) (Nicols and Heinselman, 2007; Vadas and Nicolls, 2008, 2009). At EISCAT, similar measurements are currently being prepared in the EISCAT_3D concept (Wannberg et al., 2010).

Acknowledgements. We want to thank Yasonobu Ogawa for the guidance to ESR-IPY data, Klemens Hocke for providing his $\mathrm{PhD}$ thesis and discussion on his previous AGW-TID studies, Noora Partamies and Laureline Sangali for fruitful discussions on physics of high-latitude ionosphere, and Mikko Syrjäsuo for very useful discussions on statistics. The EISCAT staff are acknowledged for their valuable work during the IPY-ESR run. EISCAT is an international Association supported by Finland (SA), Sweden (VR), Norway (NFR), United Kingdom (NERC), Japan (NIPR), China (CRIRP) and Germany (DFG). The IMAGE magnetometer data are collected as a joint European effort with FMI serving as the PI-institute. This study has been conducted with the support of the program TT-AVA and projects 115947 and 138775 by the Academy of Finland, and Federal Targeted Programme "Scientific and ScientificPedagogical Personnel of the Innovative Russia in 2009-2013” contract no 14.740.11.0742.

Topical Editor P.-L. Blelly thanks I. Horvath and another anonymous referee for their help in evaluating this paper.

\section{References}

Bristow, W. A., Greenwald, R. A., and Villain, J. P.: On the seasonal dependence of medium-scale atmospheric gravity waves in the upper atmosphere at high latitudes, J. Geophys. Res., 101, 15685-15699, 1996.

Djuth, F. T., Sulzer, M. P., Elder, J. H., and Wickwar, V. B.: Highresolution studies of atmosphere-ionosphere coupling at Arecibo Observatory, Puerto Rico, Radio Sci., 32, 2321-2344, 1997.

Hagfors, T.: Density fluctuations in a plasma in a magnetic field with applications to the ionosphere, J. Geophys. Res., 66, 16991712, 1961.

Hamming, R. W.: Digital Filters, Prentice-Hall Inc., Englewood Cliffs, New Jersey, 1977.

Hernandes-Pajares, M., Juan, M. J., and Sanz, J.: Medium-scale traveling ionospheric disturbances affecting GPS measurements: Spatial and temporal analysis, J. Geophys. Res., 111, A07S11, doi:10.1029/2005JA011474, 2006.

Hines, C. O.: Internal atmospheric gravity waves at ionospheric heights, Can. J. Phys., 38, 1441-1481, 1960.

Hocke, K.: Phase estimation with the Lomb-Scargle periodogram method, Ann. Geophys., 16, 356-358, 1998, http://www.ann-geophys.net/16/356/1998/.
Hocke, K. and Kämpfer, N.: Gap filling and noise reduction of unevenly sampled data by means of the Lomb-Scargle periodogram, Atmos. Chem. Phys., 9, 4197-4206, doi:10.5194/acp9-4197-2009, 2009.

Hocke, K. and Schlegel, K.: A review of atmospheric gravity waves and travelling ionospheric disturbances: 1982-1995, Ann. Geophys., 14, 917-940, doi:10.1007/s00585-996-0917-6, 1996.

Hocke, K., Schlegel, K., and Kirchengast, G.: Phases and amplitudes of TIDs in the high-latitude F-region observed by EISCAT, J. Atmos. Terr. Phys., 57, 245-255, 1996.

Hunsucker, R. D.: Atmospheric gravity waves generated in the high-latitude ionosphere: a review, Rev. Geophys. Space Phys., 20, 293-315, 1982.

Hunsucker, R. D.: The Sources of gravity waves, Nature, 328, 204205, 1987.

Kauristie, K., Pulkkinen, T. I., Pellinen, R. J., and Opgenoorth, H. $\mathrm{J}$.: What can we tell about global auroral-electrojet activity from a single meridional magnetometer chain?, Ann. Geophys., 14, 1177-1185, doi:10.1007/s00585-996-1177-1, 1996.

Kersley, L. and Hughes, K. A.: On the distinction between largescale and medium-scale atmospheric gravity waves, Ann. Geophys., 7, 459-462, 1989,

http://www.ann-geophys.net/7/459/1989/.

Kirchengast, G.: Elucidation of the physics of the gravity wave-TID relationship with the aid of theoretical simulations, J. Geophys. Res., 101, 13353-13368, 1996.

Kirchengast, G.: Characteristics of high-latitude TIDs from different causative mechanisms deduced by theoretical modeling, J. Geophys. Res., 102, 4597-4612, 1997.

Kirchengast, G., Hocke, K., and Schelgel, K.: Gravity waves determined by modeling of traveling ionospheric disturbances in incoherent scatter radar measurements, Radio Sci., 30, 1551-1567, 1995.

Kotake, N., Otsuka, Y., Ogawa, T., Tsugawa, T., and Saito, A.: Statistical study of medium-scale traveling ionospheric disturbances observed with the GPS networks in Southern California, Earth Planets Space, 59, 95-102, 2007.

Lanchester, B. S., Nygren, T., Jarvis, M. J., and Edwards, R.: Gravity wave parameters measured with EISCAT and Dynasonde, Ann. Geophys., 11, 925-936, 1993, http://www.ann-geophys.net/11/925/1993/.

Nicolls, M. J. and Heinselman, C. J.: Three-dimensional measurements of traveling ionospheric disturbances with the Poker Flat Incoherent Scatter Radar, Geophys. Res. Lett., 34, L21104, doi:10.1029/2007GL031506, 2007.

Nygren, T., Huuskonen, A., Lanchester, B. S., and Rishbeth, H.: Observations of tides and gravity waves in the lower thermosphere at 70 N, Adv. Space Res., 10, 10107-10110, 1992.

Ogawa, T., Nishitani, N., Otsuka, Y., Shiokawa, K., Tsugawa, T., and Hosokawa, K.: Medium-scale traveling ionospheric disturbances observed with the SuperDARN Hokkaido radar, allsky imager, and GPS network and their relation to concurrent sporadic-E irregularities, J. Geophys. Res., 114, A03316, doi:10.1029/2008JA013893, 2009.

Press, W. H. and Teukolsky, S. A.: Search Algorithm for Weak Periodic Signals in Unevenly Spaced Data, Computers in Physics, 2, 77-82, 1988.

Pryse, S. E., Kersley, L., and Walker, I. K.: Blobs and irregularities in the auroral ionosphere, J. Atmos. Terr. Phys., 58, 205-215, 
1996.

Russell, C. and McPherron, R. : Semiannual Variation of Geomagnetic Activity, J. Geophys. Res., 78, 92-108, 1973.

Saito, A., Fukao, S., and Miyazaki, S.: High resolution mapping of TEC perturbations with the GSI GPS network over Japan, Geophys. Res. Lett., 25, 3079-3082, 1998.

Scargle, J. D.: Studies in Astronomical time series analysis. II. Statistical Aspects of Spectral Analysis of Unevenly Spaced Data, Astrophysical Journal, 263, 835-853, 1982.

Shiokawa, K., Ihara, C., Otsuka, Y., and Ogawa, T.: Statistical study of nighttime medium-scale traveling ionospheric disturbances using mid-latitude airglow images, J. Geophys. Res., 108, 1052, doi:10.1029/2002JA009491, 2003.

Tsugawa, T., Saito, A., and Otsuka, Y.: A statistical study of large-scale traveling ionospheric disturbances using the GPS network in Japan, J. Geophys. Res., 109, A06302, doi:10.1029/2003JA010302, 2004.

Tsugava, T., Otsuka, Y., Coster, A. J., and Saito, A.: Medium-scale traveling ionospheric disturbances detected with dense and wide TEC maps over North America, Geophys. Res. Let., 34, L22101, doi:10.1029/2007GL031663, 2007.

Vadas, S. L. and Nicolls, M. J.: Using PFISR measurements and gravity wave dissipative theory to determine the neutral, background thermospheric winds, Geophys. Res. Lett., 35, L02105, doi:10.1029/2007GL031522, 2008.
Vadas, S. L. and Nicolls, M. J.: Temporal evolution of neutral, thermospheric winds and plasma response using PFISR measurements of gravity waves, J. Atmos. Solar-Terr. Phys., 71, 744-770, 2009.

Viereck, R.: A review of mesospheric dynamics and chemistry, Rev. Geophys., 29, 1132-1142, 1991.

Wannberg, G., Wolf., I., Vanhainen, L.-G., Koskenniemi, K., Röttger, J., Postila, M., Markkanen, J., Jacobsen, R., Stenberg, A., Larsen, R., Eliassen, S., Heck, S., and Huuskonen, A.: The EISCAT Svalbard radar: A case study in modern incoherent scatter radar system design, Radio Sci., 32, 2283-2307, 1997.

Wannberg, U. G., Andersson, H., Behlke, R., Belyey, V., Bergqvist, P., Borg, J., Brekke, A., Delsing, J., Eliasson, L., Finch, I., Grydeland, T., Gustavsson, B., Haggstrom, I., Harrison, R. A., Iinatti, T., Johansson, G., Johansson, J., Johansson, J., La Hoz, C., Laakso, T., Larsen, R., Larsmark, M., Lindgren, T., Lundberg, M., Markkanen, J., Marttala, I., McCrea, I., McKay, D., Postila, M., Puccio, W., Renkwitz, T., Turunen, E., van Eyken, A., Vanhainen, L.-G., Westman, A., and Wolf, I.: EISCAT_3D - A next-generation European radar system for upper atmosphere and geospace research, Radio Science Bulletin, 332, 75$88,2010$.

Williams, P. J. S.: Observations of Atmospheric Gravity Waves with Incoherent Scatter Radar, Adv. Space Res., 9, (5)65-(5)72, 1989. 